Voix et Images

voixetimages

\title{
Le schème organisateur chez Gabrielle Roy
}

\section{André Brochu}

Volume 14, numéro 3 (42), printemps 1989

Gabrielle Roy

URI : https://id.erudit.org/iderudit/200795ar

DOI : https://doi.org/10.7202/200795ar

Aller au sommaire du numéro

Éditeur(s)

Université du Québec à Montréal

ISSN

0318-9201 (imprimé)

1705-933X (numérique)

Découvrir la revue

Citer cet article

Brochu, A. (1989). Le schème organisateur chez Gabrielle Roy. Voix et Images, 14(3), 414-422. https://doi.org/10.7202/200795ar d'utilisation que vous pouvez consulter en ligne.

https://apropos.erudit.org/fr/usagers/politique-dutilisation/ 


\section{Le schème organisateur chez Gabrielle Roy}

\section{par André Brochu, Université de Montréal}

Dans une étude sur la Montagne secrète ${ }^{1}$, je me suis naguère attaché à dégager un schème directeur, c'est-à-dire une structure dynamique qui commandait les principaux développements du texte. Ce schème, on peut se demander s'il ne se retrouve pas dans d'autres œuvres de la romancière et s'il ne correspond pas, en somme, à une matrice permanente du vécu ou, si l'on préfere, du champ existentiel. L'ouvre serait ainsi le révélateur d'un récit fondamental, celui que l'auteur se raconte dans la plus immédiate distance à soi-même et qu'il réinvente, sous des formes diverses, tout au long de sa vie, à chaque nouvel acte de création.

Pour vérifier cette hypothèse, qui procède moins de l'approche scientifique que de l'écoute proprement critique, celle qui promeut la compréhension du texte par une démarche d'empathie, j'interrogerai deux textes, une nouvelle et un roman, dont les rédactions sont sensiblement rapprochées mais qui differrent cependant par le sujet et les thèmes.

Voici donc une nouvelle de Un jardin au bout du monde, la première, intitulée «Un vagabond frappe à notre porte». Le livre qui la contient paraît en 1975, mais la première version du «Vagabond», précise l'auteur dans sa préface, date de plus de vingt ans, donc à peu près de l'époque où paraît Alexandre Chenevert, dont je parlerai en second lieu ${ }^{2}$.

Je rappelle les principales données narratives. Un soir d'automne, quelqu'un se présente à la maison où vit Ghislaine, la narratrice, qui est alors une enfant et qui ressemble beaucoup à la Christine de Rue Deschambault. On peut penser qu'elle en est la préfiguration puisque la rédaction du recueil de nouvelles est nettement postérieure à celle du «Vagabond». La ressemblance s'étend au reste de la famille: le père de Ghislaine, Arthur Trudeau, est un sosie d'Édouard et la mère, Albertine, une sœur jumelle d'Éveline, figures dérivées de Léon et de

1 André Brochu, «La Montagne secrète: le schème organisateur», Études littéraires, vol. XVII, no 3, hiver 1984, p. 531-544; repris dans la Visée critique, Montréal, Boréal, 1988, p. 186-203.

2 En fait, «Un vagabond frappe à notre porte» a d'abord paru dans Amérique française, Montréal, janvier 1946, p. 29-51. Mais la rédaction d'Alexandre Chenevert a commencé peu après la parution de Bonheur d'occasion, signale François Ricard (Gabrielle Roy, Montréal, Fides, 1975, p. 76; et «La métamorphose d'un écrivain: essai biographique», Êtudes littéraires, vol. XVII, n 3 , hiver 1984, p. 448), à qui je dois ces renseignements. 
Mélina Roy. On retrouve, en somme, la famille des écrits semi-autobiographiques, avec des noms différents. On a l'impression curieuse, due à l'illusion rétrospective, d'un exercice de mise au point onomastique quand l'auteur fait défiler, dans la bouche du vagabond, les noms qui seront ceux des sœurs de Christine. En effet, il demande à Ghislaine: $T u$ dois être ma petite cousine Alice? (le nom rappelle, bien sûr, celui d'Alicia) puis: C'est-y Agnès alors? (JBM, p. 13). Plus loin, le nom d'Édouard est donné à un frère d'Arthur établi en Alberta.

Un homme se présente donc et il prétend être de la famille du père, un Trudeau, venu du Québec pour visiter ses cousins éloignés. Le lecteur se doute vite qu'il s'agit d'un imposteur, et la mère, femme sensée et perspicace, ne semble pas du tout partager l'enthousiasme de son mari à l'égard de l'étranger. Pourtant, elle s'abstient de formuler ses doutes, même si elle vient bien près de le faire. Est-elle sensible à la douceur, au côté chaleureux, humain du visiteur? Ou mieux, comprend-elle l'importance de ce qu'il apporte à son mari, c'est-àdire tout un passé retrouvé, une famille quittée parce qu'elle ne comportait rien d'attirant et qui maintenant se met à revivre sous des traits plus positifs?

Bien sûr, c'est un tour de force de se faire passer pour le membre d'une famille qu'on ne connaît ni d'Ève ni d'Adam, et le lecteur est tenté de taxer le récit d'invraisemblance, ou en tout cas d'accuser le père de naïveté. C'est lui qui, sans s'en rendre compte, fournit au vagabond les éléments biographiques que ce dernier, avec beaucoup d'habileté, utilise pour ses récits. D'autre part, la mère semble parfaitement lucide; mais voilà, elle se tait, et le lecteur, en quelque sorte, suit son exemple, sachant que quelqu'un, sur la scène représentative, partage son point de vue et reste prêt à intervenir.

Le vagabond a donc tôt fait de mettre le père dans sa manche, comme on dit. Il joue sur une corde sensible, le mal du pays, en ces plaines de l'Ouest où la nostalgie du Québec natal est restée très vivace. Mais la mère, elle, est beaucoup plus difficile à apprivoiser. Pourtant, à la fin, quand le père aura été détrompé par la police, qui a recueilli de nombreux témoignages incriminants, la mère se portera au secours du vagabond, allant même jusqu'à contester l'imposture. C'est qu'elle aura reconnu en lui, par delà ses pauvres subterfuges, un membre méritant de la grande famille humaine, laquelle est plus importante que la famille selon le sang qui est bafouée ici, et qu'elle lui aura pardonné en raison de la joie que véhicule son mensonge de pique-assiette, mais aussi et surtout de ce mensonge supérieur qu'est le récit. Le vagabond est l'homme des récits, celui qui raconte à chacun ses origines et qui le met en relation avec la famille universelle. De ce point de vue, il est, parmi tant d'autres dans l'œuvre de Gabrielle Roy, une figure de l'écrivain.

Où est notre schème narratif? Je dois d'abord en rappeler les composantes, telles que je les formulais dans mon analyse de la Montagne secrète.

Il nous apparaît comme la successsion de trois moments principaux:

a) une longue activité, extrêmement répétitive, visant à éliminer une quantité considérable de matière inutile pour faire apparaître un objet unique et précieux; 
b) un moment «théorique», parce que vécu dans l'inconscience et reconstitué après coup, de doute si profond que la croyance en Dieu en est ébranlée - ce moment est indispensable pour que le créateur, par exemple, réalise son dessein qui suppose, qu'il le veuille ou non, une rivalité avec l'auteur de l'univers;

c) faisant contraste avec le découragement, imprévue, la découverte éblouissante de l'objet de la quête, grain d'or ou cuvre d'art, qui reflète le sujet et le magnifie, lui apporte à la fois peine et joie (superlatives).

Le moment initial, celui de l'activité répétitive visant à isoler un élément essentiel, dans la Montagne secrète, correspond au travail du chercheur d'or qui sasse et ressasse interminablement les eaux de la rivière, ou du travail de l'artiste, Pierre, qui fait d'innombrables pochades avant de s'attaquer au grand sujet, cette montagne incomparable en laquelle il étreindra l'absolu.

Dans «Un vagabond frappe à notre porte», il correspond à ces mille et un récits - car le conteur, ici, fait beaucoup penser à Schéhérazade - par lesquels le vagabond construit la fresque familiale, avec une prudence et une habileté admirables. Un à un, les personnages du passé entrent en scène, au gré de pseudo-confidences qu'il ne faut pas précipiter (p. 22), et, bien sûr, avec la complicité inconsciente du père:

Il procédait par courtes étapes dans ses narrations, s'interrompant souvent au moment le plus pathétique ou le plus captivant, de sorte que pour entendre la fin de son histoire nous étions sans cesse disposés à lui accorder une autre journée d' hospitalité. (p. 30-31)

Le conteur procède donc par fragments, par bribes, dans la construction de la fresque familiale - un peu comme Gabrielle Roy elle-même qui, ne pouvant écrire cetté saga de la conquête de l'Ouest dont François Ricard nous a révélé le projet $^{3}$, a abordé le sujet de façon ponctuelle, discontinue, dans ses nombreuses nouvelles semi-autobiographiques. On peut penser aussi aux innombrables croquis de Pierre Cadorai reculant longtemps devant le projet de la peinture, que viendra fixer de façon plus définitive la découverte de la «montagne secrète».

Gustave - c'est le nom que s'attribue le vagabond - ne fait pas que ressusciter les membres d'une famille qu'il ne connaît que par ce qu'il en apprend de ses interlocuteurs. Il entremêle ses portraits d'évocations du pays, décrit avec minutie des fêtes de Noël et du Jour de l'An, des veillées d'hiver, des noces, et, tout d coup, Montréal, la grande ville, et tout a coup, Joliette, la petite ville ou les gens de Saint-Alphonse allaient acheter, etc. (p. 24). Bref, il donne consistance à un ensemble très varié de faits, où les véridiques font passer ceux qui sont inventés. De même, il choisit les héros de ses récits moins parmi les membres de la famille que parmi des personnages inoubliables, des êtres bizarres, cruels et obsédants et donne alors libre cours à sa verve de conteur. Il apporte l'imaginaire et peint l'homme universel, justifiant ainsi

3 François Ricard, Gabrielle Roy, Montréal, Fides, 1975, p. 112 sqq. 
l'élargissement de la scène narrative: Ah, notre parenté avec les hommes! Ou elle commence, où elle s'arrête, qui donc pourrait le dire! (p. 31)

En somme, imposteur dans son rôle de cousin, Gustave apporte une vérité d'ordre supérieur dans son rôle de conteur. En cela, il pourrait ressembler au dompteur d'ours, de Thériault, qui révolutionne tout un village en incarnant un idéal de puissance virile qu'il est bien loin de réaliser effectivement.

Mais insistons surtout sur le côté fragmentaire, discontinu du récit de Gustave, côté qui est d'ailleurs un élément important du suspense, qui est une habileté (ce Gustave, il devint très habile. Il laissait filer ses histoires. Il les morcelait en petites tranches selon une maniere d laquelle la radio plus tard nous accoutuma. Tout lui servait a les rallonger, p. 31). Le morcellement est encore accentué par le fait que tout le jour, Gustave n'est plus un conteur mais un homme à tout faire qui gagne sa croûte et qui file doux, de crainte d'être démasqué.

Le premier moment du schéma, celui de l'accumulation de gestes ou d'activités semblables, correspond donc à la narration sans cesse reprise et ajournée de la saga familiale.

Puis vient le moment où tout flanche, et c'est le moment du doute, le moment où Pierre Cadorai perd un instant le fruit de son travail et remet en question sa destinée. Il n'est pas peintre et la preuve, c'est que toutes ses toiles sont englouties par la rivière, après que son canot a chaviré.

De même, le vagabond est repris par l'appel de la route. Il était tout semblable à un grand chien maigre de notre enfance que le mauvais temps faisait entrer et qu' un pire temps invitait dehors. (p. 44) Et il disparaît, pour aller refaire ailleurs son numéro, laissant derrière lui un intérêt renouvelé pour la famille, intérêt alimenté par le rêve. Or voici que, après des années, il revient, souffrant d'une grosse fièvre. Dans son délire, il avoue implicitement son imposture en s'attribuant successivement plusieurs identités. La pluralité qui marque le premier moment du schème se réfléchit donc en cet épisode du deuxième. Et l'imposture est confirmée quand le père, Arthur, communique avec la police, qui a ouvert une enquête. Voilà donc le foisonnement des contes, des histoires, des identités, du rêve, réduit à une réalité fort sombre, l'imposture: Il n'a pas un nom, cet homme, il en a dix, vingt, autant de familles qu'il lui convient (p. 55), s'exclame le père, dont l'immense déception alimente la colère. Et c'est là que la mère entre en scène. Elle qui n'a jamais été dupe, elle veut à tout prix empêcher que le père, déçu dans son rêve, ne retombe dans une affligeante réalité: Dans les yeux de mon père, écrit la narratrice, on vit revenir le manque d' amour dans lequel si longtemps il avait dû vivre. (p. 56) L'imposture du pseudo-Gustave, une fois dénoncée, devient la blessure mortelle du père et le drame de tous ceux, autour de lui, que le rêve aide à vivre. Quand le rêve se retire, qu'il ne reste plus que la froide réalité quotidienne, c'est comme si Dieu lui-même se retirait du monde. L'écrivain, le conteur, l'artiste sont ceux par qui Dieu, ou l'art, ou le rêve, vient aux hommes.

Le troisième moment du schème sera donc la reconduction du rêve, mais un rêve qui n'est plus un mensonge, un déni de la réalité, du simple fait que ce déni est désormais voulu, choisi. Le mensonge conscient et volontaire n'est plus 
le mensonge, l'imposture acceptée n'est plus imposture mais reconnaissance de l'identité non plus selon la loi mais selon les hommes, selon la grande famille humaine. Famille que la mère incarne par excellence. On la voit dans sa cuisine, associée au spectacle d'un rayon de soleil qui [traversait la pièce] de part en part, éclairant finement la buée des plats mis à mijoter sur le poêle (p. 57). Rencontre de l'eau, du feu, de l'air, assomption poreuse d'une lumière entièrement amicale et réconfortante, maternelle. Une conjonction s'établit entre Albertine et Gustave. Enfin il leva les yeux sur ma mère. Les vieilles prunelles au regard usé brillèrent de nouveau. (p. 57) Le feu de la vie se communique à celui qui avait apporté le rêve dans une maison assaillie par les morosités de la mauvaise saison. Dans un adieu plein de charité et de gratitude, la mère salue notre cousin Gustave - et il n'est pas sûr qu'il l'entende, lui qui est déjà loin: comme Elsa, dans la Rivière sans repos, n'est pas sûre des mots que son Jimmy lui a adressés en son absence du haut de l'avion et qu'on lui a rapportés, tant bien que mal; Elsa doit se rabattre sur la sensation lumineuse des filaments de plante que son souffle fait s'élever dans l'air du soir, semblables aux cheveux dorés de son enfant - et cette sensation rappelle celle du rayon de soleil éclairant finement la buée des plats, dans le «Vagabond».

On voit que la destinée du mendiant, qui est un conteur et, à sa façon, un artiste populaire, c'est-à-dire qui tire son inspiration de ce peuple même qui est son public et qu'il raconte à lui-même, comporte les mêmes moments fondamentaux que celle de Cadorai, artiste lui aussi, et errant, et resté très près de ce peuple qu'il représente dans ses dessins quand il ne s'enfonce pas dans une exigeante solitude.

Qu'en est-il, maintenant, d'Alexandre Chenevert, petit homme sans éclat qui, malgré ses projets d'écriture - il ne vise pas au-delà des lettres aux journaux - ne saurait être considéré comme un artiste, même au sens le plus large?

On peut s'attendre à ce que le dernier moment du schéma, qui met en conjonction la réalité et le rêve dans les textes que j'ai cités, ne corresponde pas à une réussite aussi complète. Et la mort d'Alexandre, effectivement, n'a rien d'exaltant. On verra tout de même qu'elle n'est pas un échec absolu. De la même façon, les conclusions de la Montagne secrète et du "Vagabond» comportent des éléments d'ambiguitté. Pierre rejoint finalement sa montagne, il réalise son rêve de la peindre, mais il le réalise de façon tout à fait partielle et paradoxale puisque la mort l'empêche d'aller au-delà d'un simple commencement de réalisation. Il réalise son rêve pour lui, non pour les autres. Quant au vagabond, il est réhabilité par Albertine, la mère, mais il n'en est pas moins obligé de fuir, vieux et malade. Bref, le rêve est sauvé, mais la réalité reste fort cruelle pour ceux qui en sont les agents.

Mais voyons d'abord ce qu'il en est du premier moment du schème. Le début de la séquence existentielle est marqué, on le sait, par une activité 
répétitive, visant à la découverte d'un "grain d'or», d'un élément idéal qui transformera l'existence. Cet aspect répétitif, il est superbement présent dans la vie d'Alexandre. Semblables aux petits gophers des Prairies qui n'arrêtent pas de fouiller la terre de leurs pattes fines pour y enfouir leurs provisions (MS, p. 16), Alexandre est tout occupé à assurer sa survie et il vit dans une fébrilité perpétuelle. Fébrilité de la pensée d'abord, exaspérée par l'insomnie et qui le fait sauter constamment d'une idée à une autre, dans un papillottement continuel, accomplissant ainsi un tour du monde qui aboutit à un désert (p. 20). Et cette pensée est scandée par le tic-tac de l'horloge, qui est le chant même de la répétition compulsive, ennemie du repos. Celui-ci est finalement obtenu, mais au moment même où va sonner le réveil. Le repos reste un point seulement, un grain de réconfort alors qu'il devrait s'étaler dans la durée.

De même, au travail, Alexandre derrière son guichet voit défiler un à un ses clients, qui correspondent tout à fait à ce que Sartre, dans la Critique de la raison dialectique, appelle la série, cette pluralité de solitudes structurées de. façon purement extérieure. La série est à la fois continue et discontinue, elle est continue extérieurement, mais chacun de ses éléments est un cas à part, qui cependant ici ne s'écarte guère d'un certain profil: les opérations bancaires sont peu nombreuses et peu complexes. De là l'aspect fort répétitif du travail d'Alexandre, dont il ne se plaint pas, mais qui marque sa vie au coin de l'uniformité.

Or, dans cette succession mécanique des mêmes opérations, un événement, à la fois menu et considérable, se produit:

Penché sur ses papiers, Alexandre entendit tout d coup, presque indistincte a travers l'allure folle des additionneuses, s'élevant a peine au-dessus du frottement des pieds, une voix douce à son oreille, qui disait: Merci. (p. 54)

Les additionneuses, le frottement des pieds, font entendre le bruit innombrable de l'existence fonctionnelle, vouée aux opérations répétitives de la survie. Au milieu de cela, le petit mot de gratitude produit un arrêt. Il suspend la routine, instaure un temps et un espace nouveaux, événementiels, ponctuels. Deux points, en particulier, résument l'excellence de ce qui est introduit: un point de lumière d'abord - ses yeux brillaient de bienveillance (p. 54) -, une lueur du regard qui matérialise la bonté, l'ouverture de l'âme; et un point de discours puisque, à son merci, le vieux monsieur joint le nom qu'il vient de lire sur la plaque du caissier. Merci, monsieur Chenevert. Être reconnu, être salué par son nom (on sait que Alexandre Chenevert est le demier mot du roman), en toute bienveillance, voilà ce qui peut donner un sens à la vie, la mettre audessus de la répétition compulsive. Peu après cet heureux incident, on voit Alexandre retirer ses lunettes: Ses yeux apparurent à nu, des yeux gris fer, clignant a la lumière, presque sans regard et beaux cependant de leur contenu humain. Dans l'iris brillait le reflet de la lampe. (p. 54) La lumière est ici aussi associée à la manifestation de l'humain, qui est le principe même de la beauté: est beau ce qui dévoile un contenu humain, une identité pleinement vécue. 
Malgré les quelques instants de grâce auxquels aboutissent les séries particulières et qui sont les germes de ce troisième moment du schème vers lequel tend la dynamique du texte, c'est la répétition compulsive qui régit une grande partie du roman et qui génère de nombreuses séquences énumératives, par exemple la promenade nocturne où Alexandre lit les plaques des médecins, spécialistes de l'estomac, du cœur, des maladies nerveuses, des troubles de la peau, les chiropraticiens, homéopathes, dermatologues, etc.; puis la réclame des pharmacies pour divers remèdes ou médications, pénicilline, streptomycine, sérum frais gardé sur la glace, injections par voies musculaires ou intraveineuses (p. 109). Alexandre est le type même du citadin qui subit à tout moment le bombardement d'une information qui est soit celle des actualités, avec tout ce qu'elle charrie de propagande, soit celle de la publicité; et dont la conscience est peuplée de slogans obsédants, de toute une chaîne de signifiants aliénants, hallucinés. Quand, au lac Vert, Alexandre voudra exprimer dans une lettre au journal la pure joie d'exister qu'il vient de connaître pour la première fois, c'est cette batterie de formules creuses qui refera surface, impitoyablement: $L$ ' huile était principalement en Iran, Dieu se composait de trois personnes, le monde était un et indivisible... (p. 254)

Le deuxième moment du schème, c'est celui qui précède et prépare le moment merveilleux, inattendu de la satisfaction du désir fondamental. Et c'est un moment très sombre. C'est celui où Pierre Cadorai, toujours si courageux, connaît un abattement où il revit ses innombrables échecs, son «infortune» originelle. Notamment la perte, en dix circonstances diverses, du produit de sa vie: ses croquis, ses toiles. C'est le moment du doute. C'est le moment où Arthur Trudeau prend conscience d'avoir été la dupe du vagabond; et que tous les fragments de la belle fresque familiale, celle qui lui redonnait le pays des origines, sont faux. C'est le moment où le vagabond, convaincu d'imposture, perd le nom sous lequel il s'est fait connaître, et en même temps tous ses noms d'emprunt, engouffrés dans la débâcle comme les toiles de Pierre emportées par le courant.

Alexandre aussi perd son nom. D'abord sous le regard (ennuyé à l'excès) du docteur Hudon, qui

vit tout à coup en quoi cet homme l'agaçait tellement: c'était qu'il était pour ainsi dire innombrable.

Le matin, à heure fixe, il descendait de mille escaliers à la fois, courant de tous les points de la ville vers les trams archicombles. Il $s^{\prime} y$ entassait d cent, mille exemplaires. (p. 167)

Telle est, si l'on veut, la vérité d'Alexandre pour autrui, pour ce médecin compatissant qui se penche sur lui et qui découvre un être aliéné, sans identité parce que sans bonheur. Mais plus loin, c'est la maladie qui s'abat sur Alexandre et qui le rend conscient de sa dépossession. Soudain, il parut intolérable à Alexandre d'être offert pour ainsi dire à la maladie, à la mort peut-être. (p. 313) Il est alors pris au piège de sa chambre d'hôpital: Il avait l'air d'un homme emmuré qui aurait quand même, de tous côtés, cherché une 
voie possible. (p. 313) Et alors, par la fenêtre, face à la tempête déchaînée, il s'imagine un autre passé: être allé au pôle Sud avec Scott ou Amundsen [...] Il aurait voulu s'approprier les belles vies (p. 314) La rêverie d'Alexandre devant la tempête qui suscite et disperse à mesure ses fantasmes, ses images idéales du moi, se superpose au délire du vagabond malade qui laisse filer ses identités, au regard navré de Pierre devant le tourbillon des eaux qui engloutit ses œuvres.

La souffrance arrache aussi à Alexandre une protestation souveraine contre un Dieu qui a inventé de faire souffrir (p. 379); elle rejoint le sentiment de Pierre, que sa vie est privée de sens.

Le dernier moment du schème, dans Alexandre Chenevert, c'est celui de la mort. Tout le roman nous mène vers cette irréductible catastrophe. J'ai signalé déjà qu'elle était sous-jacente à l'histoire de Pierre Cadorai, qui trouve enfin sa montagne au prix de sa vie. Elle se lit en filigrane dans le départ du cousin Gustave, qui ne peut plus espérer recommencer sa joyeuse imposture dans la petite colonie francophone du Manitoba. Il restera l'homme du rêve et du récit, le porteur de fiction, lui-même estompé dans la légende du souvenir.

Tel sera aussi Alexandre. Sa survie, certes, semble assez mince:

Cependant, ailleurs que dans les églises, il arrive encore aujourd' hui, après ces quelques années, que le nom soit prononcé - et n'est-ce point chose mystérieuse et tendre, qu'à ce nom corresponde un lien?... Il arrive qu' ici et là, dans la ville, quelqu' un dise:

\section{- ...Alexandre Chenevert... (p. 384)}

Le nom, c'est un lien, une mise en relation du présent et du passé, des vivants et des morts, de l'accidentel qui est le réel avec le nécessaire, qui est le rêve. Par la mort, Alexandre devient un symbole de douceur et de tendresse, il devient l'homme même:

Dans les derniers moments, une telle douceur avait touché ce visage que les témoins se persuadaient avec ce mourant que la seule assurance, sur terre, vient de notre déraisonnable tendresse humaine. (p. 384)

La mort réalise, entre Alexandre et les êtres humains, une communion qu'il avait rêvée toute sa vie, sans pouvoir la réaliser autrement que dans la solitude la plus extrême. Cette solitude, qui ramène aux origines, c'est celle du lac Vert, qui est la montagne secrète d'Alexandre, et il ne la retrouve, comme Pierre Cadorai, que dans l'illumination finale, dans ce moment où tout le corps sombre et où ne reste plus que le cœur.

C'est le moment du repos - du repos éternel, sans doute, mais d'un repos ardemment désiré depuis cette inlassable, cette compulsive activité répétitive par laquelle l'artiste, comme le vagabond, comme le caissier, assurent leur survie, espérant toujours qu'elle fera place à la vie véritable, cette vie qui est le rêve, sous toutes ses formes: art, lac Vert ou île du Sud, famille des origines comme dans le Québec lointain ou la Petite-Poule-d'Eau, Jimmy revenu honorer cavalièrement, du haut du ciel, sa mère éperdue d'un tel bonheur. À la 
lumière de ce schème, on peut se demander si la destinée de Florentine Lacasse, qu'on a souvent interprétée - moi le premier ${ }^{4}$ - en termes très négatifs, la réalité supplantant brutalement le rêve comme Emmanuel succède à Jean Lévesque, ne connaît pas une conclusion plus heureuse. Certes, le réalisme succède à la passion après cette nuit d'enfer, chez Marguerite, où le cœur de Florentine était devenu comme insensible (BO, p. 271). Mais Florentine aboutit à un repos qui n'est pas sans rappeler celui d'Alexandre, après les agitations de sa petite vie: Le calme qui l'enveloppait lui était aussi bienfaisant, après le bouleversement des derniers mois, qu'un banc au soleil à qui a marché des nuits et des nuits. (BO, p. 383)

Belle image d'un bonheur d'occasion, ce banc dans le plein jour, qui récompense la marche interminable et nocturne d'une existence. La lumiere est au bout du livre, dans un repos qui est la mort et qui est la vraie vie, qui est la vie tout humaine, tout âme, et amen.

4 André Brochu, «La structure sémantique de Bonheur d'occasion», Revue des sciences humaines de Lille, no 173, 1979, p. 37-47; repris dans la Visée critique, Montréal, Boréal, 1988, p. 169-185. 